\title{
The effect of human GATA4 gene mutations on the activity of target gonadal promoters
}

\author{
Marie France Bouchard ${ }^{1,2 \star}$, Hiroaki Taniguchi ${ }^{1,2 \star}$ and Robert S Viger ${ }^{1,2,3}$ \\ ${ }^{1}$ Reproduction, Perinatal and Child Health, Centre de Recherche du Centre Hospitalier Universitaire de Québec (CRCHUQ), Room T1-49, 2705 Laurier Boulevard, \\ Québec City, Québec, Canada G1V 4G2 \\ ${ }^{2}$ Centre de Recherche en Biologie de la Reproduction (CRBR), Université Laval, Québec City, Québec, Canada \\ ${ }^{3}$ Department of Obstetrics and Gynecology, Faculty of Medicine, Université Laval, Québec City, Québec, Canada \\ (Correspondence should be addressed to R S Viger; Email: robert.viger@crchul.ulaval.ca) \\ *(M F Bouchard and H Taniguchi contributed equally to this work)
}

\begin{abstract}
GATA transcription factors are crucial regulators of cell-specific gene expression in many tissues including the gonads. Although clinical cases of reproductive dysfunction have yet to be formally linked to GATA gene mutations, they have begun to be reported in other systems. Heterozygous GATA4 mutations have been associated with cases of congenital heart defects. Little is known, however, about the effect of these mutations on gonadal gene transcription. Since individuals carrying these mutations do not appear to suffer from gross reproductive defects, we hypothesized that this might be due to the differential transcriptional properties of the mutant proteins on heart versus gonadal target genes. Five mutations (S52F, E215D, G295S, V266M, and E359X) were recreated in the rat GATA4 protein. Several parameters were used to analyze the transcriptional properties of the mutants: activation of known gonadal target promoters (Star, Cyp19a1, and Inha), DNA binding, and interaction with GATA4 transcriptional partners. Three mutations (S52F, G295S, and E359X) reduced GATA4 transcriptional activity on the different gonadal promoters. With the exception of the G295S mutant, which showed a significant loss of DNA-binding affinity, the decrease in activity of the other GATA4 mutants was not associated with a change in DNA binding. All GATA4 mutants retained their ability to interact and cooperate with their major gonadal partners (NR5A1 and NR5A2) thereby compensating in part for the loss in intrinsic GATA4 transcriptional activity. Thus, unlike the heart, where the GATA4 mutations have deleterious effects, our data suggest that they would have a lesser impact on gonadal gene transcription and function.
\end{abstract}

Journal of Molecular Endocrinology (2009) 42, 149-160

\section{Introduction}

GATA4 is a member of the GATA family of zinc finger DNA-binding proteins that recognizes the consensus DNA motif (A/T) GATA $(\mathrm{A} / \mathrm{G})$ in the promoter region of target genes (Molkentin 2000). In the reproductive tract, GATA4 is prominently expressed in somatic cells of both the testis and ovary, where it has been shown and/or proposed to regulate the transcription of numerous target genes (Viger et al. 2008). These targets include genes coding for hormones or components of the steroidogenic pathway such as anti-Müllerian hormone $(A m h)$, inhibin subunit $\alpha(\operatorname{Inh} a)$, steroidogenic acute regulatory protein (Star), $\mathrm{P} 450$ aromatase (Cyp19a1), P450 side chain cleavage (Cyp11a1), P450 $17 \alpha$-hydroxylase $/ 17,20$ lyase (Cyp17a1), and $3 \beta$ hydroxysteroid dehydrogenase $/ \Delta^{5}-\Delta^{4}$ isomerase type 2 (HSD3B2; Viger et al. 2008). In gonadal cells, GATA4 activity has been shown to be modulated via cooperative interactions with other transcriptional regulators and/or cofactors (Viger et al. 2008). These include the nuclear receptors steroidogenic factor 1 (SF-1/NR5A1) and liver receptor homolog 1 (LRH-1/NR5A2), which cooperate with GATA4 to enhance transcription from the promoters of many of the GATA4 targets listed above (Viger et al. 2008). GATA4 transcriptional activity can also be modulated by post-translational modifications such as phosphorylation (Viger et al. 2008). Activation of the cAMP/PKA signaling pathway is a major mechanism for conveying the hormone responsiveness of many gonadal genes (Richards 1994). GATA4 can be directly phosphorylated by PKA resulting in a dramatic enhancement of its transactivation potential on many GATA4-dependent gonadal promoters (Tremblay et al. 2002, Tremblay \& Viger 2003a).

While human GATA mutations affecting normal reproductive function have yet to be identified, GATA gene sequence variations have begun to be linked to human diseases in other systems. For example, GATA1 mutations have been reported in inherited anemia and thrombocytopenia (Nichols et al. 2000), as well as in Down's syndrome-related acute leukemia (Wechsler 
et al. 2002). Although no mutation of the GATA2 gene has yet been demonstrated in hematological diseases, it has been reported that decreased GATA2 expression is observed in CD34 positive cells in patients with aplastic anemia (Fujimaki et al. 2001). GATA3 mutations have been associated with HDR syndrome, a genetic disease characterized by hypoparathyroidism, sensorineural deafness, and renal dysplasia (Van Esch et al. 2000). GATA6 frameshift mutations in the DNA-binding domain and C-terminus have been reported to cause malignant astrocytomas (Kamnasaran et al. 2007).

There have now been reports of several GATA4 gene mutations affecting cardiac development and function (Garg et al. 2003, Okubo et al. 2004, Hirayama-Yamada et al. 2005, Nemer et al. 2006, Tang et al. 2006). Most of these mutations occur in amino acid residues highly conserved across species ranging from zebrafish to human, underlying the relative importance of these residues for proper GATA4 function (the characteristics of some of these GATA4 mutations are summarized in Table 1). A missense mutation, G296S, causes a decrease in GATA4 transcriptional activity by weakening its DNAbinding capacity and by disrupting the cooperative interaction with its transcriptional partner TBX5, ultimately leading to reduced expression of target genes in the developing heart (Garg et al. 2003). Another missense mutation, E216D, also causes a reduction in GATA4 transcriptional activity although the DNAbinding ability of the protein and its physical (proteinprotein) and functional (transcriptional) interaction with Friend of GATA 2 (FOG2) on cardiac targets are not apparently affected (Nemer et al. 2006). A third mutant, S52F, has also been reported to reduce GATA4 transactivation potential, although its DNA-binding capacity remains intact (Hirayama-Yamada et al. 2005). Similarly, two frameshift mutations, causing the loss of a large portion of the C-terminal end of GATA4, S358X (Okubo et al. 2004), and E359X (Garg et al. 2003), appear to be transcriptionally inactive although the molecular interactions with DNA and/or other proteins have not been investigated. Additional GATA4mutations have been described: V267M and V380M amino acid substitutions of GATA4 protein have also been related to heart defects (Tang et al. 2006), but the molecular basis for the cardiac phenotypes have yet to be established.

To date, human GATA4 mutations have been identified based on their deleterious effects on heart development and function. The impact of these mutations on GATA4 function outside of the cardiac system has not been investigated. This includes the reproductive tract where GATA4 has been shown to play a critical role in early gonadal morphogenesis, at least in the mouse (Tevosian et al. 2002, Bouma et al. 2007, Manuylov et al. 2007). In the present study, we have recreated five human GATA4 mutations (listed in Table 1), known to affect heart function, in the rodent protein and tested their functional properties - DNA-binding capacity, transactivation potential, physical and functional interaction with known regulatory partners - on a variety of GATA4dependent gonadal promoters. Our results show that GATA4-dependent activation of different gonadal promoters can be significantly affected by some of these mutations. However, the different GATA4 mutants retained their ability to interact and cooperate with their major gonadal partners thereby compensating in part for the loss in intrinsic GATA4 transcriptional activity. Thus, unlike the heart where these GATA4 mutations have major deleterious effects, our data suggest that they would have less dramatic consequences on gonadal gene transcription and function.

\section{Materials and methods}

\section{Plasmids}

The murine -902 bp (Star), - 218 bp PII aromatase (Cyp19a1), and -679 bp inhibin $\alpha$ (Inha) promoter constructs have been described previously (Tremblay \& Viger 2001). The wild-type GATA4 expression vector has

Table 1 Characteristics of the GATA4 mutations studied

\begin{tabular}{|c|c|c|c|c|c|c|}
\hline & $\begin{array}{l}\text { Rodent } \\
\text { equivalent }\end{array}$ & $\begin{array}{l}\text { Nucleotide } \\
\text { change }\end{array}$ & $\begin{array}{l}\text { Gene } \\
\text { region }\end{array}$ & $\begin{array}{l}\text { Protein } \\
\text { region }\end{array}$ & $\begin{array}{l}\text { Associated human } \\
\text { defect(s) }\end{array}$ & References \\
\hline \multicolumn{7}{|l|}{ Mutation } \\
\hline E216D & E215D & c. $648 \mathrm{C}>\mathrm{G}$ & Exon 3 & NZf region & TOF & Nemer et al. (2006) \\
\hline V267M & V266M & c. $799 \mathrm{G}>\mathrm{A}$ & Exon 4 & CZf region & CHD & Tang et al. (2006) \\
\hline $\begin{array}{l}\text { E359X (frame- } \\
\text { shift truncation) }\end{array}$ & $\begin{array}{l}\text { E359X (rat) } \\
\text { E360X (mouse) }\end{array}$ & c. 1075 delG & Exon 6 & C-ter & ASD, dextrocardia & Garg et al. (2003) \\
\hline
\end{tabular}

AR, aortic regurgitation; ASD, atrial septal defect; AVSD, atrioventricular septal defect; CHD, congenital heart defect; CZf, C-terminal zinc finger; MR, mitral regurgitation; NLS, nuclear localization signal; NZf, N-terminal zinc finger; PDA, patent ductus arteriosus; PS, pulmonary stenosis; PVS, pulmonary valve stenosis; VSD, ventricular septal defect; TAD1, transcriptional activation domain 1; TOF, tetralogy of Fallot. 
also been reported previously (Tremblay et al. 2001). The different GATA4 human mutations were recreated using the rat GATA4 coding sequence by site-directed mutagenesis. The mutations were introduced according to the manufacturer's instructions using a QuikChange XL mutagenesis kit (Stratagene, La Jolla, CA, USA) and the oligonucleotide primers indicated in Table 2. Expression plasmids for mouse NR5A1 (Lala et al. 1992), NR5A2 (Galarneau et al. 1996), and PKA catalytic subunit (Mayr \& Montminy 2001) were kindly provided by Drs Keith Parker (University of Texas Southwestern Medical Center, Dallas, TX, USA), Luc Bélanger (Centre de recherche en cancérologie, Centre de recherche du CHUQ, Université Laval, Québec City, Canada), and Marc Montminy (The Salk Institute for Biological Studies, La Jolla, CA, USA) respectively.

\section{Cell culture and transfections}

African green monkey kidney CV-1 cells and mouse TM3 Leydig cells were used for all transfection studies. As a heterologous line (not expressing GATA or NR5A factors), CV-1 cells are a very convenient line for comparing the transcriptional properties of the different GATA4 mutants presented herein. TM3 cells express endogenous GATA proteins and are representative of a gonadal somatic cell line (Tremblay \& Viger 2001). CV-1 cells were grown in DMEM supplemented with $10 \%$ newborn calf serum. TM3 cells were cultured in a 1:1 mixture of Ham's F12 and DMEM containing $5 \%$ horse serum and $2.5 \%$ FBS. HeLa cells, used to overexpress the different GATA4 mutants for western analysis, were grown in DMEM/F12 supplemented with $10 \%$ fetal bovine serum. All transfections were done in 24-well plates using the calcium phosphate precipitation method (Chen \& Okayama 1987). Cells were plated at an initial density of $2 \times 10^{4}$ cells/well and transfected $24 \mathrm{~h}$ later. Culture medium was changed $12-16 \mathrm{~h}$ after transfection, and the cells were finally harvested the following day by lysing the cells in $50 \mu$ lysis buffer
(100 mM Tris-HCl (pH 7.9), 0.5\% Igepal (SigmaAldrich Oakville, Canada), and $5 \mathrm{mM}$ dithiothreitol). An aliquot of the lysate was then assayed for luciferase activity using a Luminoskan Ascent microplate luminometer (Fisher Scientific Limited, Ottawa, Canada) and luciferine (BD Biosciences, Mississauga, Canada) as substrate. In all experiments, the total amount of DNA was kept constant at $2 \mu \mathrm{g}$ /well using Sp64 (Promega Madison, WI, USA) as carrier DNA. Data reported represent the average of at least three experiments (range of 3-15), each done in duplicate.

\section{Nuclear extracts and western blot}

Nuclear extracts were prepared by the procedure outlined by Schreiber et al. (1989). In western analyses, $30 \mu \mathrm{g}$ aliquots of nuclear extracts from HeLa cells overexpressing GATA4 (wild-type or mutated) were separated by SDS-PAGE then electrotransferred to Hybond polyvinylidene difluoride membrane (GE Healthcare Life Sciences, Baie d'Urfé, Canada). GATA4 proteins were detected using a commercially available GATA4 polyclonal antibody (\# sc-1237X, Santa Cruz Biotechnology, Santa Cruz, CA, USA) and a VECTASTAIN-ABC-Amp Western blot detection kit (Vector Laboratories Canada, Burlington, Canada).

\section{Electrophoretic mobility shift assay}

Recombinant GATA4 wild-type and mutated proteins were in vitro translated using a QuickCoupled TNT in vitro transcription and translation system (Promega). Electrophoretic mobility shift assays (EMSA; DNAbinding) were performed using a ${ }^{32}$ P-labeled doublestranded oligonucleotide corresponding to the conserved GATA element of the proximal murine Star promoter (Tremblay \& Viger 2001). The oligos used were (GATA site is italicized) - sense: $5^{\prime}$-GATCCACTTTT TTATCTCAAGTGA-3', antisense: $5^{\prime}$ - GATCTCACTTGAGATAAAAAAGTG-3'). Binding reactions

Table 2 Primers used to generate the different GATA4 mutants

\section{Sense (S)/antisense (A)}

\section{Mutation}

S52F

E215D

V266M

G295S

E359X

S
$A$
S
$A$
S
$A$
S
$A$
S
A

\section{Sequence $^{a}$}

The position of the deletion is indicated by an asterisk $\left({ }^{*}\right)$.

${ }^{a}$ Mutated nucleotides are italicized. 
were done using $2 \mu \mathrm{l}$ of in vitro translated protein in $20 \mu \mathrm{l}$ buffer $(4 \mathrm{mM}$ Tris-HCl ( $\mathrm{pH} 7 \cdot 9), 24 \mathrm{mM} \mathrm{KCl}$, $0.4 \mathrm{mM}$ EDTA (pH 8.0), $0.4 \mathrm{mM}$ dithiothreitol, $5 \mathrm{mM}$ $\mathrm{MgCl}_{2}, 10 \%$ glycerol, and $100 \mathrm{ng}$ polydeoxyinosinicdeoxycytidylic acid) for $1 \mathrm{~h}$ at $4{ }^{\circ} \mathrm{C}$. GATA4-containing complexes were subsequently analyzed by electrophoresis through a $4 \%$ non-denaturing polyacrylamide gel in $0.5 \times$ Tris-borate-EDTA buffer at $200 \mathrm{~V}$ for $1.5 \mathrm{~h}$ at $4{ }^{\circ} \mathrm{C}$ followed by autoradiography.

\section{Production of histidine fusion proteins}

Recombinant histidine (HIS)-NR5A1 and HIS-NR5A2 fusion proteins were obtained by cloning the corresponding coding sequences in frame with HIS using the commercially available pRSETB fusion protein vector (Invitrogen, Burlington, Canada). The resulting constructs were introduced into the Escherichia coli strain BL21, and the fusion proteins were produced by inducing the bacterial cultures with isopropyl-1-thio-D-galactopyranoside. After the induction, the bacterial cultures were lysed by sonication and the fusion proteins were purified using a Talon metal affinity resin (BD Biosciences) according to instructions outlined by the manufacturer.

\section{In vitro pull-down (protein-protein interaction) assay}

Protein-protein interaction studies were done using ${ }^{35}$ S-labeled in vitro translated wild-type or mutated GATA4 proteins, and the purified HIS-NR5A1, HISNR5A2, or HIS- $\beta$-galactosidase (HIS-LacZ) fusion proteins coupled to a Talon metal affinity resin (BD Biosciences). The ${ }^{35}$ S-labeled GATA4 proteins were obtained using the TNT system from Promega. The pull-down procedure has been described previously (Martin et al. 2005).

\section{Statistical analysis}

Statistical comparisons between multiple groups (Figs $2 \mathrm{~A}, 4 \mathrm{~A}$ and $\mathrm{B}$, and $5 \mathrm{~A}$ and $\mathrm{B}$ ) were analyzed by one-way ANOVA followed by a Student-Newman-Keuls test. Where normality and/or equal variance among groups was not met, data were analyzed with equivalent (Kruskal-Wallis ANOVA/Student-Newman-Keuls) non-parametric tests. $P<0.05$ was considered significant. All statistical analyses were done with the aid of the SigmaStat 3.5 software package (Systat Software Inc., Point Richmond, CA, USA).

\section{Results}

\section{The GATA4 mutants have reduced transcriptional activity on gonadal promoters}

To investigate, the effect of human GATA4 mutations reported to affect heart development and function on the transcriptional regulation of gonadal target genes; we recreated five mutations in context of the highly conserved rat orthologue (Fig. 1 and Table 1). The different GATA4 mutants were first compared with the wild-type protein for their ability to transactivate three known GATA-responsive gonadal promoters (Star, Cyp19a1, and Inha) in heterologous CV-1 fibroblasts that lack endogenous GATA4 expression and TM3 mouse Leydig cells that express endogenous GATA proteins (Fig. 2A). Much like their action on heart-specific promoters (Garg et al. 2003, Okubo et al. 2004, Hirayama-Yamada et al. 2005, Nemer et al. 2006, Tang et al. 2006), the GATA4 G295S, E359X, and S52F mutations also markedly reduced the ability of the GATA4 protein to transactivate the three gonadal promoters in CV-1 cells (Fig. 2A). This effect was intrinsic to the mutant proteins since the mutations themselves did not affect the expression level or the cellular localization of the overexpressed proteins (Fig. 2B). In contrast to these three mutations however, the E215D and V266M mutants retained significant transcriptional activity (Fig. 2A). For the E215D mutant, its transactivation potential was equivalent to the wild-type GATA4 protein. Similar results were also observed in TM3 cells; transcriptional activities were correspondingly lower, however, due to the presence of existing GATA factors in these cells.

\section{DNA-binding affinity of the GATA4 mutants}

The reduced transcriptional activity observed for some of the GATA4 mutants suggested that the amino acid modifications involved might directly affect GATA4 DNA binding. To study this possibility, DNA binding of wild-type and mutant GATA4 proteins on the conserved GATA element of the murine Star promoter was compared by EMSA (Fig. 3). Consistent with the initial study characterizing the binding of the mouse orthologue of the human G296S GATA4 mutant protein on a heart-specific promoter GATA element (Garg et al. 2003), the rodent G295S mutant also showed markedly reduced binding compared with the wild-type GATA4 protein on a typical gonadal promoter GATA element (Fig. 3A, left panel). Weak binding of the G295S mutant was still observed even at a fivefold excess of protein (Fig. 3A, right panel), suggesting that impaired DNA binding contributes to the reduced transcriptional activity of this particular mutant. In contrast to the G295S variant, the other GATA4 mutants (E215D, S52F, V266M, and E359X) showed strong DNA binding reminiscent of the wild-type protein (Fig. 3B).

\section{PKA-dependent enhancement of GATA4-mediated transactivation}

We have previously reported that the transcriptional activity of GATA4 in gonadal cells is enhanced by 


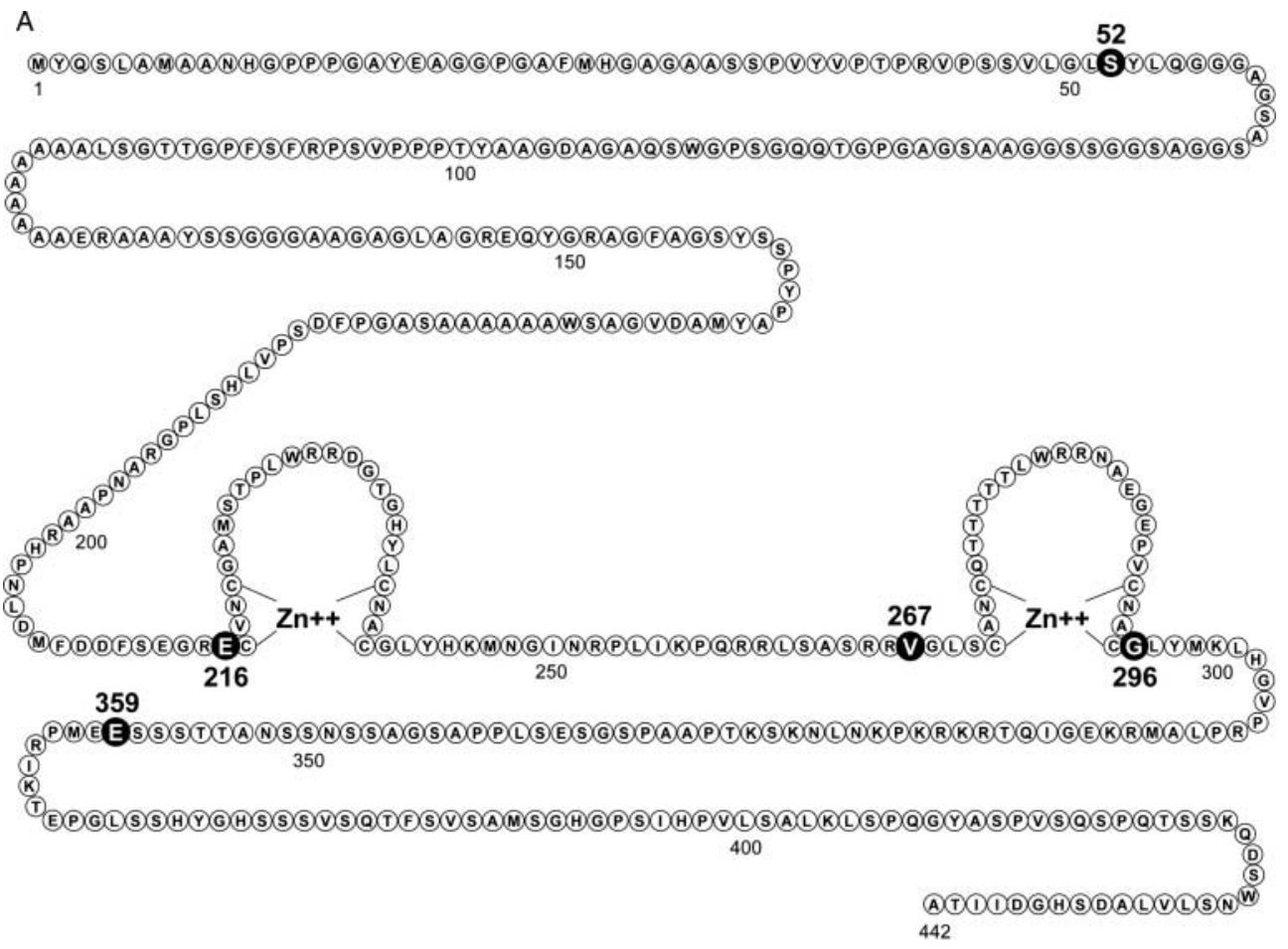

B

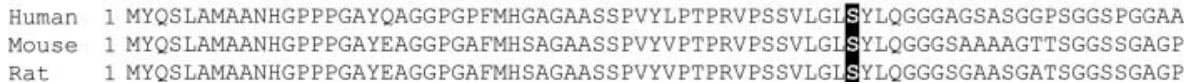

Human SGAGPGTQQGSPGWSQAGATGAAYTPPPVSPRESFPGTTGSLAAAAAAAAAREAAAYSSGGGAAGAGLAGREQYG Mouse SGAGPGTQQGSPGWSQAGAEGAAYTPPPVSPRFSFPGTTGSLAAAAAAAAAREAAAYGSGGGAAGAGLAGREQYG Rat SGAGPGTQQGSPGWSQAGAEGAAYTPPPVSPRFSFPGTTGSLAAAAAAAAAREAAAYSSSGGAAGAGLAGREQYG

Human RAGEAGSYSSPYPAYMADVGASWAAAAAASAGPFDSPVLHSLPGRANPAARHPNLDMFDDESEGE ECVNCGAMST Mouse RPGEAGSYSSPYPAYMADVGASWAAAAAASAGPFDSPVLHSLPGRANPG-RHPNLDMFDDFSEGRECVNCGAMST Rat RPGEAGSYSSPYPAYMADVGASWAAAAAASAGPFDSPVLHSLPGRANPA-RHPNLDMFDDFSEGRECVNCGAMST

Human PLWRRDGTGHYLCNACGLYHKMNGINRPLIKPQRRLSASRR $\mathbf{V}$ GLSCANCQTTTTTLWRRNAEGEPVCNACGLYMK Mouse PLWRRDGTGHYLCNACGLYHKMNGINRPLIKPQRRLSASRRVGLSCANCQTTTTTLWRRNAEGEPVCNACGLYMK Rat PLWRRDGTGHYLCNACGLYHKMNGINRPLIKPQRRLSASRRVGLSCANCQTTTTTLWRRNAEGEPVCNACELYMK

Human LHGVPRPLAMRKEGIQTRKRKPKNLNKSKTPAAPSGSESLPPASGASS-NSSNATTSSS- - EREMRPRIKTEPGLSS Mouse LHGVPRPLAMRKEGIQTRKRKPKNLNKSKTPAGPAG-ETLPPSSGASSGNSSNATSSSSSSEEMRPIKTEPGLSS Rat LHGVPRPLAMRKEGIQTRKRKPKNLNKSKTPAGPPG-ESLPPSSGASS-NSSNATSSSSSSEEMRPIKTEPGLSS TTGTAAPCPRRSQSVRCLAMGPPSTLSSRP

Human HYGHSSSVSQTESVSAMSGHGPSIHPVLSALKLSPQGYASPVSQSPQTSSKQDSWNSLVLADSHGDIITA 442 Mouse HYGHSSSMSQTFST--VSGHGPSIHPVLSALKLSPQGYASPVTQTSQASSKQDSWNSLVLADSHGDIITA 441

Rat HYGHSSSMSQTFST--VSGHGSSIHPVLSALKLSPQGYPSPVTQTSQASSKQDSWNSLVLADSHGDIITA 440

Figure 1 Amino acid positions of five known GATA4 heterozygous point mutations associated with human disease. (A) Structure and amino acid sequence of the human GATA4 protein. The locations of the various mutations recreated in this study (S52, E216, V267, G296, and E359) are indicated by black circles. (B) The mutated GATA4 amino acid residues (black boxes) are conserved between human and rodent species. The E359X mutation produces a frameshift truncation; the modified sequence is indicated in bold italic, above and downstream of the mutation. The rodent equivalents of the different GATA4 human mutations are provided in Table 1.

cAMP/PKA-mediated phosphorylation and that this enhancement is an important mechanism for up-regulating expression of certain hormone-dependent gonadal target genes (Tremblay et al. 2002, Tremblay \& Viger $2003 a$ ). We therefore surmised that mutations in GATA4 might impair its ability to respond to PKA stimulation. To test this possibility, PKA catalytic subunit was co-transfected with wild-type GATA4 or the different GATA4 mutants (Fig. 4). PKA enhancement of GATA4 activity was assessed on both the Star (Fig. 4A) and Cyp19a1 (Fig. 4B) promoters. As expected, PKA markedly enhanced wild-type GATA4 


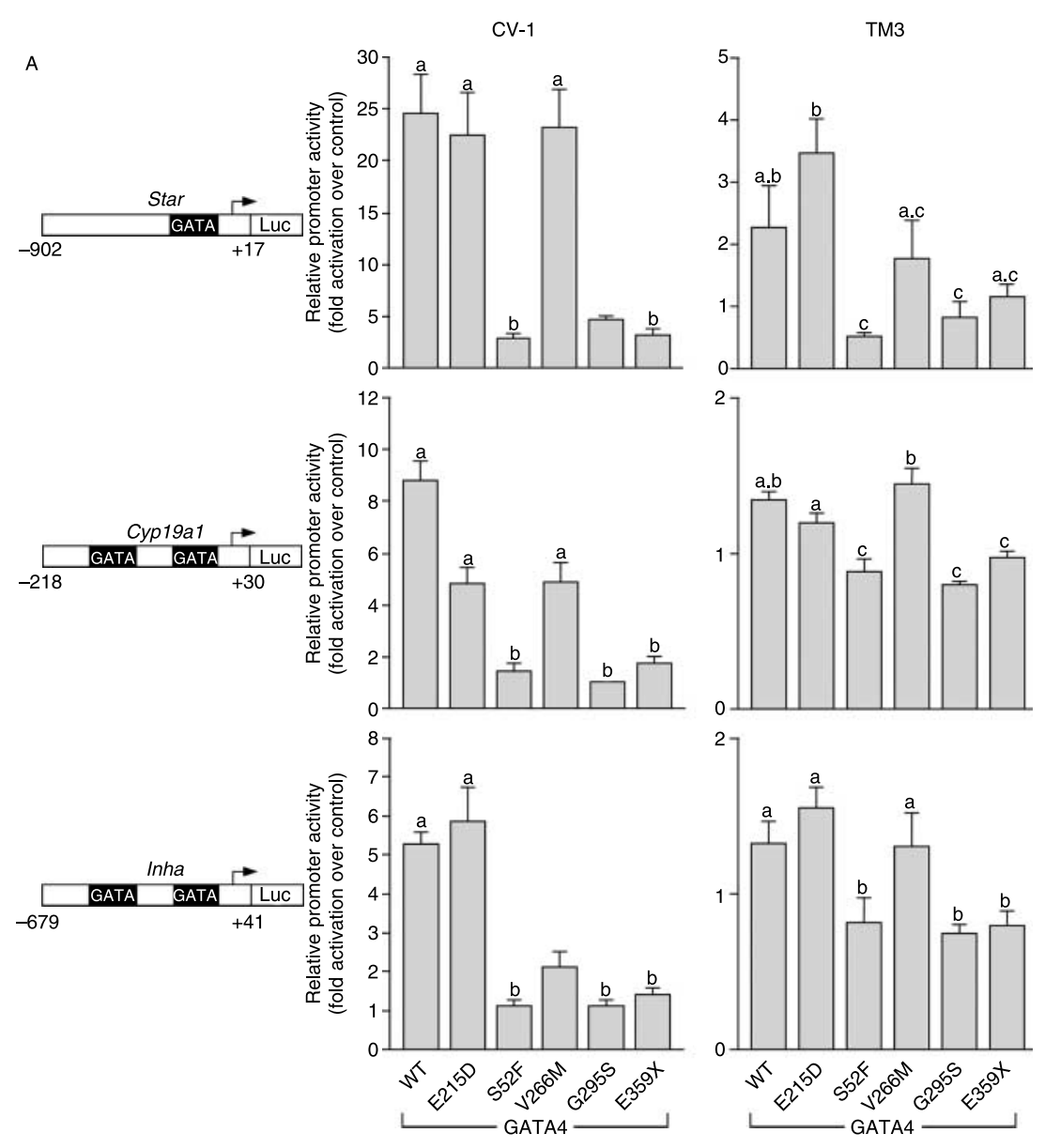

B

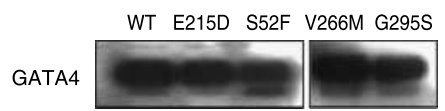

Figure 2 The various GATA4 mutations differentially affect the transcriptional properties of the GATA4 protein on different gonadal promoters. (A) The ability of the wild-type (WT) and mutant GATA4 proteins to transactivate the murine Star, aromatase (Cyp19a1), and inhibin $\alpha$ (Inha) promoters when overexpressed in CV-1 fibroblasts or TM3 mouse Leydig cells. For each cell line, cells were co-transfected with the indicated luciferase reporters along with either an empty expression vector (serving as control) or expression vectors (50 $\mathrm{ng}$ ) for the different GATA4 proteins as indicated. All promoter activities are reported as fold activation over control ( \pm S.E.M). Groups with similar letters are not significantly different from one another $(P>0 \cdot 05)$. (B) HeLa cells were transfected with expression vectors encoding WT GATA4 or the different mutants. Western blot analysis shows that all proteins were similarly expressed in isolated nuclear extracts.

activity on both promoters in both CV-1 and TM3 cells. Interestingly, PKA overexpression still resulted in a significant enhancement of the activity of most, if not all, of all the GATA4 mutant proteins (Fig. 4A and B). For some of the mutants, PKA-mediated enhancement was even greater than the wild-type GATA4 protein. In CV-1 cells, this was particularly evident for the S52F, G295S, and E359X proteins where transcriptional activity in the absence of PKA was very low. Moreover, PKA was able to raise the activity of these three mutants to the level of the unstimulated wild-type GATA4 protein.

\section{Functional cooperation between GATA4 and its transcriptional partners NR5A1 and NR5A2}

The nuclear receptor NR5A1 has been shown to be an important transcriptional partner for GATA factors in enhancing the promoter activities of several genes normally expressed in endocrine cells (Viger et al. 2008). More recent studies have extended these findings to include the related factor NR5A2 (Bouchard et al. 2005, Martin et al. 2005, Robert et al. 2006). We therefore tested whether the different 

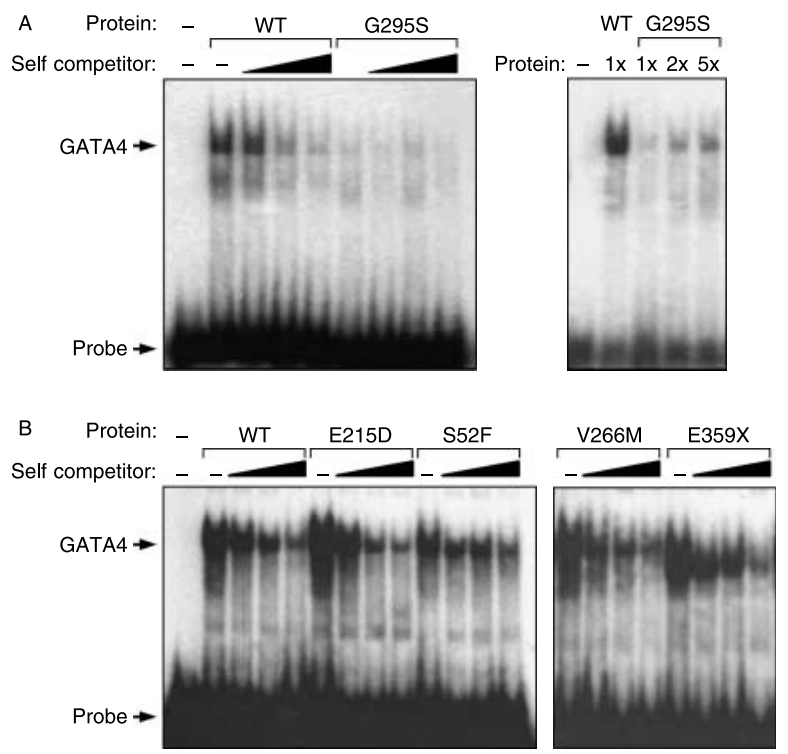

Figure 3 DNA-binding properties of the GATA4 mutants. Electrophoretic mobility shift assays were used to assess the binding of in vitro produced WT or mutated GATA4 proteins to a double-stranded ${ }^{32} \mathrm{P}$-labeled oligonucleotide corresponding to the consensus GATA element (nucleotide position - $63 \mathrm{bp}$ ) of the murine Starpromoter. (A) Left panel: the G295S mutation reduces DNA binding. Binding of GATA4 WT and G295S mutant proteins are competed with increasing doses (solid triangle) of unlabeled oligonucleotides (molar excesses of $2 \times, 5 \times$, and $10 \times$ ). Right panel: the GATA4 G295S mutant shows decreased DNA binding, even at a concentration that is five times higher than that of the WT GATA4 protein. (B) The other GATA4 mutants (E215D, S52F, V266M, and E359X) exhibit DNA-binding capacities similar to the WT GATA protein. The solid triangles indicate increasing amounts $(2 \times, 5 \times$, and $10 \times)$ of unlabeled (self) competitor oligonucleotides.

GATA4 mutations could disrupt the functional synergism between GATA4 and NR5A1 (Fig. 5A) or between GATA4 and NR5A2 (Fig. 5B) on the Cyp19a1 promoter. The Cyp19a1 promoter is a well-characterized target for GATA4 and NR5A1 or NR5A2 (Tremblay \& Viger 2001, Clyne et al. 2002, 2004, Bouchard et al. 2005). As expected, NR5A1 and NR5A2 strongly synergized with wild-type GATA4 in both CV-1 and TM3 cells (Fig. 5A and B). In CV-1 cells, synergism was still observed with four out of the five GATA4 mutants with NR5A1 and all mutants with NR5A2. A similar pattern was observed in TM3 cells except for the S52F, G295S, and E359X mutants. Interestingly, the hG296S mutant that was originally shown to be unable to interact with its cardiac-specific partner TBX5 (Garg et al. 2003), maintained its ability to functionally cooperate with its partners in the context of gonadal gene transcription, at least in CV-1 cells.

The functional cooperation between GATA4 and NR5A factors requires that the proteins contact each other via a direct protein-protein interaction (Bouchard et al. 2005, Martin et al. 2005). In vitro pull-down assays were used to assess whether the different GATA4 mutants could still physically interact with either NR5A1 or NR5A2 (Fig. 5C). In these experiments, NR5A1 and NR5A2 are expressed as HIS-tagged fusion proteins immobilized to a metal resin. The different GATA4 proteins are then expressed as ${ }^{35}$ S-labeled proteins. Labeled protein retained on the resin indicates an interaction which is visualized on an acrylamide gel. As shown in Fig. 5C (upper panels), both the wild-type and G295S GATA4 proteins were retained by the NR5A fusion proteins. A HIS-LacZ fusion protein was used as a non-specific control. Similar experiments were carried out with the remaining GATA4 mutants that showed interactions similar to the wild-type protein (Fig. 5C, lower panel).

\section{Discussion}

Initial in vitro work characterizing GATA4 target genes in gonadal cells (LaVoie 2003, Tremblay \& Viger 2003b, Viger et al. 2004), along with subsequent genetic studies in the mouse (Tevosian et al. 2002, Bouma et al. 2007, Manuylov et al. 2007), has provided strong evidence that the GATA4 transcription factor plays a central role in reproductive function. Despite the lack of direct genetic evidence, the expectation is that this critical role extends to human reproductive function. The paucity of data in the literature describing GATA4 mutations associated with human disease is not unexpected given the early developmental roles that have been ascribed to this factor (Kuo et al. 1997, Molkentin et al. 1997). This notwithstanding, heterozygous GATA4 mutations have been associated with several cases of congenital heart defects (Garg et al. 2003, Okubo et al. 2004, Hirayama-Yamada et al. 2005, Nemer et al. 2006, Tang et al. 2006). Little is known, however, about the molecular basis for these heart phenotypes and even further whether these mutations could compromise GATA4 function in a gonadal context. In the present study, we report the effect of five different human GATA4 mutations (S52F, E216D, V267, G296S, and E359X) known to affect heart function on the transcriptional properties of GATA4 on target gonadal promoters.

To study the different mutations, GATA4 mutants were recreated in the rat orthologous protein. At the outset, it is unlikely that these mutations have a major detrimental effect in the reproductive organs, as they do in the heart, since individuals carrying these mutations appear to be able to procreate (Garg et al. 2003, Okubo et al. 2004, Hirayama-Yamada et al. 2005, Nemer et al. 2006, Tang et al. 2006). Absence of gross phenotypic abnormalities, however, does not necessarily preclude an effect at the molecular level. Indeed, in our analysis of the mutations, three GATA4 mutants 

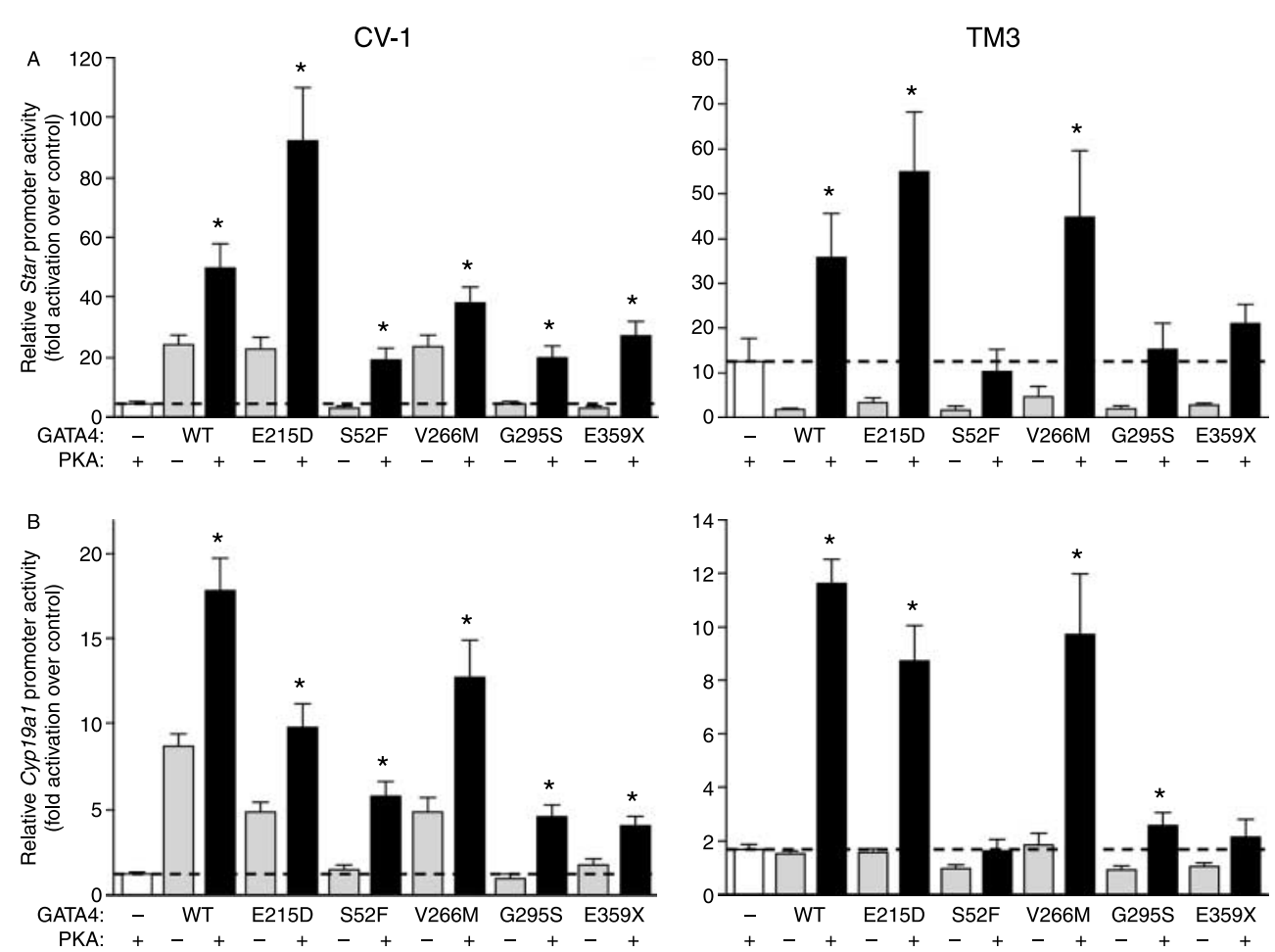

Figure 4 The ability of PKA to enhance the transcriptional properties of the GATA4 mutants on different gonadal promoters. CV-1 and TM3 cells were co-transfected with either a Star (A) or Cyp19a1 (B) luciferase reporter along with either an empty expression vector (control) or expression vectors (50 ng) for WT or mutated GATA4 in the presence or absence of PKA catalytic subunit (50 ng). All promoter activities are reported as fold activation over control ( \pm S.E.M). Promoter activity stimulated by PKA alone is indicated by the dashed line. For each GATA4 protein tested, an asterisk $\left(^{*}\right)$ denotes a statistically significant difference $(P<0.05)$ between the stimulated group ( + PKA) and both the unstimulated group ( $-\mathrm{PKA})$ and PKA alone.

(S52F, G295S, and E359X) exhibited markedly weak transcriptional activity, compared with the wild-type protein, on three separate gonadal promoters (Fig. 2A). This effect is similar to what has been demonstrated on cardiac-specific promoters (Garg et al. 2003, Schluterman et al. 2007). The drop in transactivation potential associated with these mutants was not simply due to the amount of protein present, or to impaired cellular localization, since the GATA4 mutants and wild-type protein were expressed at comparable levels in HeLa cell nuclear extracts (Fig. 2B). Interestingly, the G295S mutant was the only one to show a dramatic reduction in DNA-binding affinity on a gonadal GATA promoter element even when used at concentrations five times that of the wild-type protein (Fig. 3A). These findings are consistent with the previously reported data in the heart (Garg et al. 2003), and suggest that the G295S mutant would poorly activate gonadal promoters due to a decrease in DNA binding. The role of the E359X variant remains to be better defined since it is still unclear whether the protein is properly and stably translated in an in vivo context. Based on our in vitro data, we can assume that the 44 nonsense amino acids added to the $\mathrm{C}$-terminal domain of the prematurely truncated GATA4 protein adversely affects its function. The S52F mutant had decreased transcriptional activity (Fig. 2A), but fully retained its DNA-binding capacity (Fig. 3B). Similarly, Schluterman et al. (2007) recently showed that the S52F mutation had decreased transcriptional activity on the heart-specific $A N F$ and $\alpha-M H C$ promoters. They suggested that the mutation at position 52 altered a serine residue within the GATA4 $\mathrm{N}$-terminal activation domain thereby disrupting the function of this region by changing its structure. Thus, conformational changes induced by the mutations likely explain the reduced transcriptional activity of some of the mutant proteins on both cardiac and gonadal target promoters.

Interestingly, the GATA4 E215D mutant had the same level of activity as the wild-type protein on gonadal promoters (Fig. 2A). This is different from the heart where the same mutant had reduced transcriptional activity on the $A N F$ promoter without changes in DNAbinding ability or cellular localization of the protein (Nemer et al. 2006). These results are not a complete surprise since an engineered GATA4 mutation (E215K), targeting the same amino acid and leading to the loss of interaction between GATA4 and FOG2, also retained 

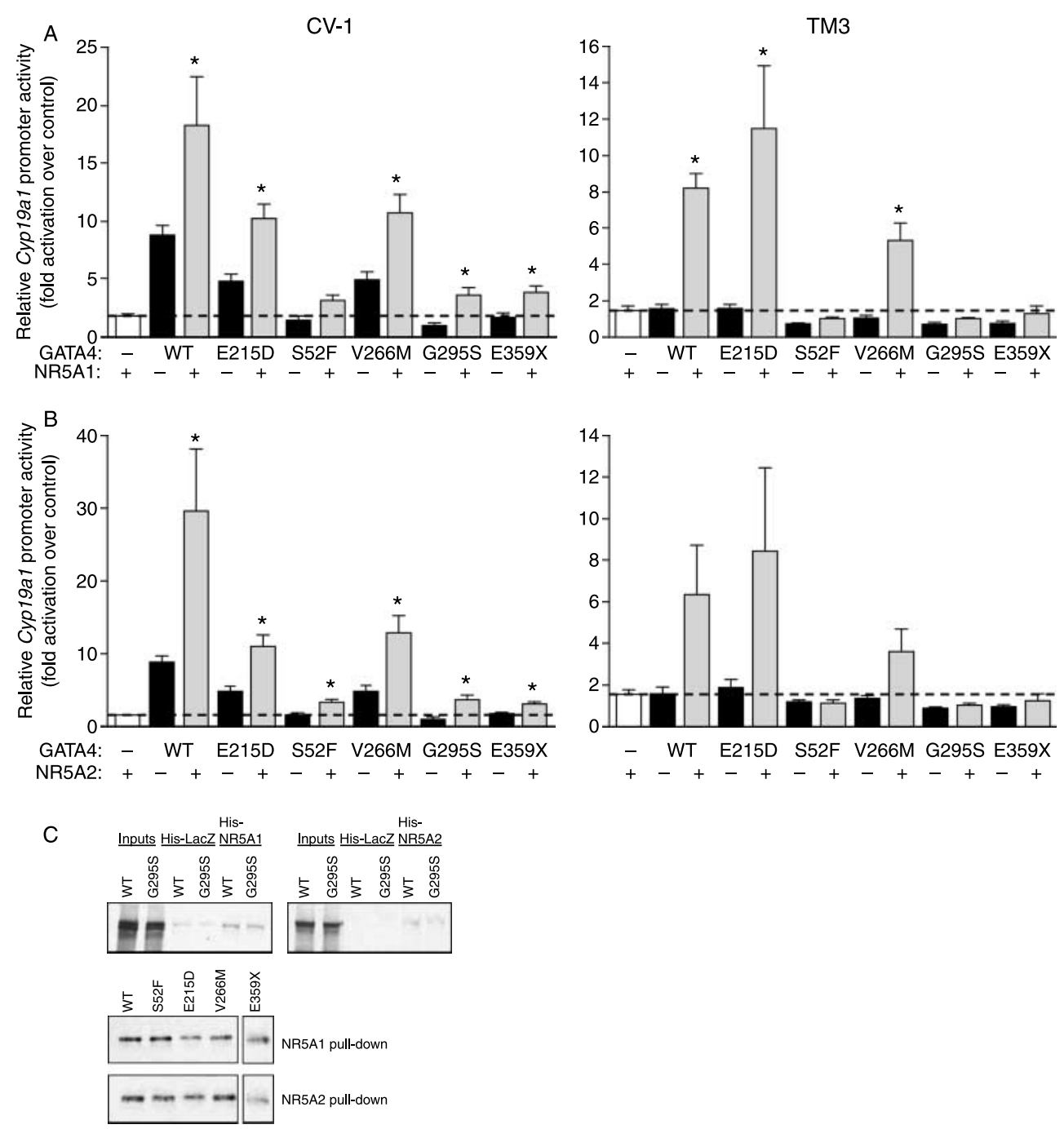

Figure 5 The ability of NR5A family members to transcriptionally cooperate and physically interact with the GATA4 mutants. (A) Cooperation with NR5A1. (B) Cooperation with NR5A2. CV-1 and TM3 cells were co-transfected with the Cyp19a1 luciferase reporter (which contains binding sites for both GATA and NR5A proteins) along with either an empty expression vector (control) or different combinations of expression vectors for WT or mutated GATA4 (50 ng), NR5A1 (25 ng), and NR5A2 (100 ng) as indicated. All promoter activities are reported as fold activation over control. Promoter activity stimulated by NR5A1 or NR5A2 alone is indicated by the dashed line. For each GATA4 protein tested, an asterisk $\left({ }^{*}\right)$ denotes a statistically significant difference $(P<0.05)$ between the synergism group (gray bar; GATA4 and NR5A factors both present) and groups where the GATA4 (black bar) and NR5A (open bar) factors were used alone. (C) In vitro pull-down assays were used to demonstrate that a physical protein-protein interaction between NR5A factors and GATA4 is retained with the different GATA4 mutants. Input indicates $10 \%$ of the labeled protein used in the pull-down assay.

strong transcriptional activity on different gonadal promoters (Robert et al. 2002). The differential activation elicited by the E215D mutant is then most likely due to differences in promoter context. Differential promoter sensitivity was also observed in the present study where the V266M mutant fully activated the Star promoter but had reduced activity on the Cyp19a1 and Inha promoters (Fig. 2A). Therefore, with respect to the E215D mutant, important differences in the way the mutated protein behaves at a molecular (i.e., transcriptional) level might be the reason why the heart, from a phenotypic standpoint, is more sensitive to the E215D mutation.

The E216D, V266M, and G295S mutations are located in close vicinity to the GATA4 zinc finger domains that are essential for DNA binding (Fig. 1A). The zinc finger region of GATA proteins, including GATA4, also mediates important protein-protein 
interactions with other transcriptional regulators that help define the proper spatiotemporal expression of target genes (Molkentin 2000, Viger et al. 2008). Indeed, in the heart, Garg et al. showed that the probable molecular basis for the pronounced cardiac phenotype associated with the G295S mutation involved a failed protein-protein interaction with TBX5, a transcription factor responsible for normal heart development and function (Garg et al. 2003). In endocrine cells, we and others have shown that the nuclear receptors NR5A1 and NR5A2 are important transcriptional partners for GATA4 (Viger et al. 2008). Although NR5A1 is expressed in the heart, at least at the transcript level (Ramayya et al. 1997, Nishimura et al. 2004), there is currently no evidence for a functional role for this receptor as a transcriptional partner for GATA4 in this tissue. Unlike TBX5 in the heart (Garg et al. 2003), the functional synergism between the G295S mutant and its gonadal partners NR5A1 and NR5A2 was not compromised (Fig. 5A and B). The G295S mutant likely retains its ability to transcriptionally cooperate with NR5A factors because it can still physically interact with the two receptors (Fig. 5C). Similar to the G295S mutant, the E215D variant could also transcriptionally cooperate with NR5A1 and NR5A2 (Fig. 5). Since this amino acid substitution does not impair the interaction of GATA4 with FOG2 (Nemer et al. 2006), the E215 residue is likely important for the recruitment of a yet unidentified transcriptional partner, essential for proper heart tube formation, but not necessarily required for gonadal development and function. Thus, again, the observation that the mutant GATA4 proteins partially retained some of their transcriptional properties on target gonadal promoters provides some molecular basis as to why these mutations do not appear to cause any pronounced phenotypic defects in the reproductive tract but do so in the heart. It also stresses the importance of specific protein-protein interactions for the regulation of GATA4 activity in different cellular contexts. In contrast to the gonads, the heart might simply be more sensitive to disruptions in GATA4 function.

Another possibility for the lack of reproductive phenotypes associated with reported GATA4 mutations might be redundancy. Sertoli cells of the testis, for example, express multiple GATA factors including GATA1, GATA4, and GATA6 (Viger et al. 2004). In this case, loss of GATA4 function might be compensated by either GATA1 or GATA6. The likelihood of compensatory mechanisms for GATA function in the gonads is supported by at least one genetic study in the mouse which showed that disruption of GATAl function in Sertoli cells produces no major gonadal defect, most likely due to compensation by other GATA family members (Lindeboom et al. 2003). Yet another possibility is the fact that although some of the
GATA4 mutants exhibited a significant reduction in transcriptional activity on different gonadal target (Fig. 2A), many of these proteins showed a marked enhancement of activity in the presence of PKA to levels approaching or even surpassing the unstimulated wildtype protein (Fig. 4).

Taken together, our data suggest that the human GATA4 mutations that have been associated with severe congenital heart abnormalities would likely have less dramatic consequences on gonad-specific gene transcription, since the mutant proteins retain many of their transcriptional properties on different GATA4dependent gonadal target promoters. During the course of this study, more germ line heterozygous GATA4 mutations have since been reported (Rajagopal et al. 2007, Reamon-Buettner et al. 2007, Tomita-Mitchell et al. 2007). Interestingly, some of these occur in the GATA4 intronic sequences or the $3^{\prime}$ non-translated region of the GATA4 mRNA. These specific mutations leave the coding sequence unaltered and have been predicted to affect RNA folding, probably leading to its decay (Reamon-Buettner et al. 2007). Thus, far, only fully penetrant germ line GATA4 mutations have been reported as a result of screening large families where congenital heart defects are present in more than one generation. Recently, the screening of large cohorts of individuals presenting cardiac malformations uncovered the first non-fully penetrant GATA4 mutations (Rajagopal et al. 2007, Tomita-Mitchell et al. 2007). Given the identification of these novel GATA4 mutations, a similar screen of individuals presenting reproductive abnormalities, and especially that involving gonadal sex reversal is both warranted and needed.

\section{Declaration of interest}

There is no conflict of interest that could be perceived as prejudicing the impartiality of the research reported.

\section{Funding}

R S Viger is holder of the Canada Research Chair in Reproduction and Sex Development. This work was supported by a grant from the Canadian Institutes of Health Research (CIHR) to R S Viger.

\section{Acknowledgements}

We thank Drs Keith Parker (SF-1 expression vector), Luc Bélanger (LRH-1 expression vector), and Marc Montminy (PKA catalytic $\alpha$ subunit expression vector) for generously providing plasmids used in this study. 


\section{References}

Bouchard MF, Taniguchi H \& Viger RS 2005 Protein kinase A-dependent synergism between GATA factors and the nuclear receptor, liver receptor homolog-1, regulates human aromatase (CYP19) PII promoter activity in breast cancer cells. Endocrinology $1464905-4916$.

Bouma GJ, Washburn LL, Albrecht KH \& Eicher EM 2007 Correct dosage of Fog2 and Gata4 transcription factors is critical for fetal testis development in mice. PNAS 104 14994-14999.

Chen C \& Okayama H 1987 High efficiency transformation of mammalian cells by plasmid DNA. Molecular and Cellular Biology 7 2745-2752.

Clyne CD, Speed CJ, Zhou J \& Simpson ER 2002 Liver receptor homologue-1 (LRH-1) regulates expression of aromatase in preadipocytes. Journal of Biological Chemistry 277 20591-20597.

Clyne CD, Kovacic A, Speed CJ, Zhou J, Pezzi V \& Simpson ER 2004 Regulation of aromatase expression by the nuclear receptor LRH-1 in adipose tissue. Molecular and Cellular Endocrinology 215 39-44.

Van Esch H, Groenen P, Nesbit MA, Schuffenhauer S, Lichtner P, Vanderlinden G, Hardling B, Beetz R, Bilous RW, Holdaway I et al. 2000 GATA3 haplo-insufficiency causes human HDR syndrome. Nature 406 419-422.

Fujimaki S, Harigae H, Sugawara T, Takasawa N, Sasaki T \& Kaku M 2001 Decreased expression of transcription factor GATA-2 in haematopoietic stem cells in patients with aplastic anaemia. British Journal of Haematology 113 52-57.

Galarneau L, Pare JF, Allard D, Hamel D, Levesque L, Tugwood JD, Green S \& Belanger L 1996 The alphal-fetoprotein locus is activated by a nuclear receptor of the Drosophila FTZ-F1 family. Molecular and Cellular Biology 16 3853-3865.

Garg V, Kathiriya IS, Barnes R, Schluterman MK, King IN, Butler CA, Rothrock CR, Eapen RS, Hirayama-Yamada K, Joo K et al. 2003 GATA4 mutations cause human congenital heart defects and reveal an interaction with TBX5. Nature 424 443-447.

Hirayama-Yamada K, Kamisago M, Akimoto K, Aotsuka H, Nakamura Y, Tomita H, Furutani M, Imamura S, Takao A, Nakazawa M et al. 2005 Phenotypes with GATA4 or NKX2.5 mutations in familial atrial septal defect. American Journal of Medical Genetics. Part A 135 47-52.

Kamnasaran D, Qian B, Hawkins C, Stanford WL \& Guha A 2007 GATA6 is an astrocytoma tumor suppressor gene identified by gene trapping of mouse glioma model. PNAS 104 8053-8058.

Kuo CT, Morrisey EE, Anandappa R, Sigrist K, Lu MM, Parmacek MS, Soudais C \& Leiden JM 1997 GATA-4 transcription factor is required for ventral morphogenesis and heart tube formation. Genes and Development 11 1048-1060.

Lala DS, Rice DA \& Parker KL 1992 Steroidogenic factor 1, a key regulator of steroidogenic enzyme expression, is the mouse homolog of fushi tarazu-factor I. Molecular Endocrinology 6 $1249-1258$.

LaVoie HA 2003 The role of GATA in mammalian reproduction. Experimental Biology and Medicine 228 1282-1290.

Lindeboom F, Gillemans N, Karis A, Jaegle M, Meijer D, Grosveld F \& Philipsen S 2003 A tissue-specific knockout reveals that Gatal is not essential for Sertoli cell function in the mouse. Nucleic Acids Research 31 5405-5412.

Manuylov NL, Fujiwara Y, Adameyko II, Poulat F \& Tevosian SG 2007 The regulation of Sox9 gene expression by the GATA4/FOG2 transcriptional complex in dominant $\mathrm{XX}$ sex reversal mouse models. Developmental Biology 307 356-367.

Martin LJ, Taniguchi H, Robert NM, Simard J, Tremblay JJ \& Viger RS 2005 GATA factors and the nuclear receptors, steroidogenic factor $1 /$ liver receptor homolog 1 , are key mutual partners in the regulation of the human 3 3 -hydroxysteroid dehydrogenase type 2 promoter. Molecular Endocrinology 19 2358-2370.

Mayr B \& Montminy M 2001 Transcriptional regulation by the phosphorylation-dependent factor CREB. Nature Reviews. Molecular Cell Biology 2 599-609.
Molkentin JD 2000 The zinc finger-containing transcription factors GATA-4, -5 , and -6 . Ubiquitously expressed regulators of tissuespecific gene expression. Journal of Biological Chemistry 275 38949-38952.

Molkentin JD, Lin Q, Duncan SA \& Olson EN 1997 Requirement of the transcription factor GATA-4 for heart tube formation and ventral morphogenesis. Genes and Development 11 1061-1072.

Nemer G, Fadlalah F, Usta J, Nemer M, Dbaibo G, Obeid M \& Bitar F 2006 A novel mutation in the GATA4 gene in patients with tetralogy of Fallot. Human Mutation 27 293-294.

Nichols KE, Crispino JD, Poncz M, White JG, Orkin SH, Maris JM \& Weiss MJ 2000 Familial dyserythropoietic anaemia and thrombocytopenia due to an inherited mutation in GATA1. Nature Genetics 24 266-270.

Nishimura M, Naito S \& Yokoi T 2004 Tissue-specific mRNA expression profiles of human nuclear receptor subfamilies. Drug Metabolism and Pharmacokinetics 19 135-149.

Okubo A, Miyoshi O, Baba K, Takagi M, Tsukamoto K, Kinoshita A, Yoshiura K, Kishino T, Ohta T, Niikawa N et al. 2004 A novel GATA4 mutation completely segregated with atrial septal defect in a large Japanese family. Journal of Medical Genetics 41 e97.

Rajagopal SK, Ma Q, Obler D, Shen J, Manichaikul A, Tomita-Mitchell A, Boardman K, Briggs C, Garg V, Srivastava D et al. 2007 Spectrum of heart disease associated with murine and human GATA4 mutation. Journal of Molecular and Cellular Cardiology 43 677-685.

Ramayya MS, Zhou J, Kino T, Segars JH, Bondy CA \& Chrousos GP 1997 Steroidogenic factor 1 messenger ribonucleic acid expression in steroidogenic and nonsteroidogenic human tissues: Northern blot and in situ hybridization studies. Journal of Clinical Endocrinology and Metabolism 82 1799-1806.

Reamon-Buettner SM, Cho SH \& Borlak J 2007 Mutations in the 3'untranslated region of GATA4 as molecular hotspots for congenital heart disease (CHD). BMC Medical Genetics 838.

Richards JS 1994 Hormonal control of gene expression in the ovary. Endocrine Reviews 15 725-751.

Robert NM, Tremblay JJ \& Viger RS 2002 FOG-1 and FOG-2 differentially repress the GATA-dependent activity of multiple gonadal promoters. Endocrinology 143 3963-3973.

Robert NM, Miyamoto Y, Taniguchi H \& Viger RS 2006 LRH-1/NR5A2 cooperates with GATA factors to regulate inhibin alpha-subunit promoter activity. Molecular and Cellular Endocrinology 257-258 $65-74$.

Sarkozy A, Conti E, Neri C, D'Agostino R, Digilio MC, Esposito G, Toscano A, Marino B, Pizzuti A \& Dallapiccola B 2005 Spectrum of atrial septal defects associated with mutations of NKX2.5 and GATA4 transcription factors. Journal of Medical Genetics 42 e16.

Schluterman MK, Krysiak AE, Kathiriya IS, Abate N, Chandalia M, Srivastava D \& Garg V 2007 Screening and biochemical analysis of GATA4 sequence variations identified in patients with congenital heart disease. American Journal of Medical Genetics. Part A 143 817-823.

Schreiber E, Matthias P, Muller MM \& Schaffner W 1989 Rapid detection of octamer binding proteins with 'mini-extracts', prepared from a small number of cells. Nucleic Acids Research 176419.

Tang ZH, Xia L, Chang W, Li H, Shen F, Liu JY, Wang Q \& Liu MG 2006 Two novel missense mutations of GATA4 gene in Chinese patients with sporadic congenital heart defects. Chinese Journal of Medical Genetics 23 134-137.

Tevosian SG, Albrecht KH, Crispino JD, Fujiwara Y, Eicher EM \& Orkin SH 2002 Gonadal differentiation, sex determination and normal Sry expression in mice require direct interaction between transcription partners GATA4 and FOG2. Development 129 $4627-4634$.

Tomita-Mitchell A, Maslen CL, Morris CD, Garg V \& Goldmuntz E 2007 GATA4 sequence variants in patients with congenital heart disease. Journal of Medical Genetics 44 779-783. 
Tremblay JJ \& Viger RS 2001 GATA factors differentially activate multiple gonadal promoters through conserved GATA regulatory elements. Endocrinology 142 977-986.

Tremblay JJ \& Viger RS $2003 a$ Transcription factor GATA-4 is activated by phosphorylation of serine 261 via the cAMP/PKA pathway in gonadal cells. Journal of Biological Chemistry 278 22128-22135.

Tremblay JJ \& Viger RS $2003 b$ Novel roles for GATA transcription factors in the regulation of steroidogenesis. Journal of Steroid Biochemistry and Molecular Biology 85 291-298.

Tremblay JJ, Robert NM \& Viger RS 2001 Modulation of endogenous GATA-4 activity reveals its dual contribution to Müllerian inhibiting substance gene transcription in Sertoli cells. Molecular Endocrinology 15 1636-1650.

Tremblay JJ, Hamel F \& Viger RS 2002 Protein kinase A-dependent cooperation between GATA and C/EBP transcription factors regulates StAR promoter activity. Endocrinology 143 3935-3945.
Viger RS, Taniguchi H, Robert NM \& Tremblay JJ 2004 Role of the GATA family of transcription factors in andrology. Journal of Andrology 25 441-452.

Viger RS, Mazaud Guittot S, Anttonen M, Wilson DB \& Heikinheimo M 2008 Role of the GATA family of transcription factors in endocrine development, function, and disease. Molecular Endocrinology 22 781-798.

Wechsler J, Greene M, McDevitt MA, Anastasi J, Karp JE, Le Beau MM \& Crispino JD 2002 Acquired mutations in GATAl in the megakaryoblastic leukemia of Down syndrome. Nature Genetics 32 $148-152$.

Received in final form 30 October 2008

Accepted 13 November 2008

Made available online as an Accepted Preprint 13 November 2008 